\title{
A case involving the spontaneous reduction of an ameloblastic fibroma
}

\author{
Mekaru Yasuaki ${ }^{1 *}$, Ueda Gousei ${ }^{1}$, Higa Tsutomu ${ }^{2}$, Kouchi Masato², \\ Tatetsu Masaharu' ${ }^{3}$, Irei Mitsutaka ${ }^{1}$
}

CrossMark

\section{Abstract}

Objective: Ameloblastic fibroma is an uncommon type of mixed odontogenic tumor. It is caused by the growth of the odontogenic epithelium and mesenchymal tissue and can have adverse effects on the formation of dental structures. It usually arises in the mandible, but cases involving the mandibular ramus are rare.

Methods: Here, we report a case of ameloblastic fibroma in the right mandibular ramus involving a 15 -year-old male patient. According to panoramic x-ray examinations, the tumor shrank spontaneously in the 3 years before surgery (from approximately $80 \mathrm{~mm} \times 55 \mathrm{~mm}$ to $50 \mathrm{~mm} \times 25 \mathrm{~mm})$.

Results: A histological evaluation revealed the proliferation of odontogenic epithelial tissue and mesenchymal elements. The Ki-67 index of the lesion was $0 \%$.

Conclusion: Cases of ameloblastic fibroma involving patients aged $\geq 22$ years old are very likely to recur and undergo malignant changes.
${ }^{1}$ Department of Dentistry, Oral and Maxillofacial Surgery, Okinawa Prefectural Chubu Hospital, Okinawa, Japan

${ }^{2}$ Department of Dentistry, Oral and Maxillofacial Surgery, Okinawa Prefectural Nanbu Medical Center \& Children's Medical Center, Okinawa, Japan

${ }^{3}$ Department of Dentistry, Oral and Maxillofacial Surgery, Okinawa Prefectural Miyako Hospital, Japan

*Corresponding to: Mekaru Yasuaki, Department of Dentistry, Oral and Maxillofacial Surgery, Okinawa Prefectural Chubu Hospital, Okinawa, Japan panabia2008@live.jp

Received: 6 November 2017 Revised: 14 November 2017 Accepted: 15 November 2017 Available online: 1 December 2017

Keywords: Ameloblastic fibroma, Odontogenic tumor, Mandibular ramus

Cite this Article: Yasuaki M, Gousei U, Tsutomu H, Masato K, Masaharu T, Mitsutaka I. 2017. A case involving the spontaneous reduction of an ameloblastic fibroma. Journal of Dentomaxillofacial Science 2(3): 194-196. D0I: 10.15562/jdmfs.v2i3.640

\section{Introduction}

Ameloblastic fibroma (AF) is an uncommon type of mixed odontogenic tumor. ${ }^{1,2}$ It usually presents as a slow-growing swelling. Most cases are asymptomatic and are discovered incidentally (as unilocular radiolucent lesions) during routine oral examinations. ${ }^{3}$ We report a case of AF in the mandibular ramus involving a 15 year old male patient. According to panoramic $\mathrm{x}$-ray examinations, the tumor shrank spontaneouslyin the 3 years before surgery (from approximately $80 \mathrm{~mm} \times 55 \mathrm{~mm}$ to $50 \mathrm{~mm} \times 25 \mathrm{~mm}$ ). The patient is still being followed-up.

\section{Case Report}

A 15 year old male was referred to the Department of Dentistry Oral and Maxillofacial Surgery Okinawa Prefectural Chubu Hospital to have a radiolucent lesion in his right ramus evaluated. The same location had become inflamed twice before, and oral administration antibiotics were administered on each occasion.

A physical examination revealed a painless mild swelling in his right cheek. Paralysis of the chin nerve field was absent. An intra-oral examination detected a painless swelling extending from the posterior region to the ascending ramus of the right mandible. The swollen region was not tender. The right lower third molar was clinically absent.
A panoramic radiograph showed a unilocular radiolucent lesion with poorly demarcated borders measuring approximately $50 \mathrm{~mm} \times 25 \mathrm{~mm}$ in the molar/ramus area of the right mandible. The third molar was located above it figure 1.

A computed tomography scan showed a mass in the right ramus figure 2. It included the crown of the third molar. Although moderate bulging of the right mandibular ramus (compared with the left mandibular ramus) was observed, no perforation or pressure absorption of the mandibular cortical bone was seen.

Based on the patient's signs and symptoms, a provisional diagnosis of odontogenic cyst or a benign tumor was made.

In mid-March 2015, the patient underwent enucleation under general anesthesia via an intraoral approach figure $3 \mathrm{~A}$. The impacted third molar within the lesion was extracted during surgery figure 3B.

Interestingly, a postoperative panoramic x-ray obtained in December 2012 showed agiant permeable lesion (measuring approximately $80 \mathrm{~mm} \times 55 \mathrm{~mm}$ ) that occupied the whole mandibular ramus figure 4 . Thus, the lesion had shrunk during the 3 years before surgery.

A histological evaluation revealed the proliferation of odontogenic epithelial tissue and mesenchymal elements figure 5A-B. The Ki-67 index of the lesion was $0 \%$ figur $5 \mathrm{C}$. A diagnosis of AF was made. 


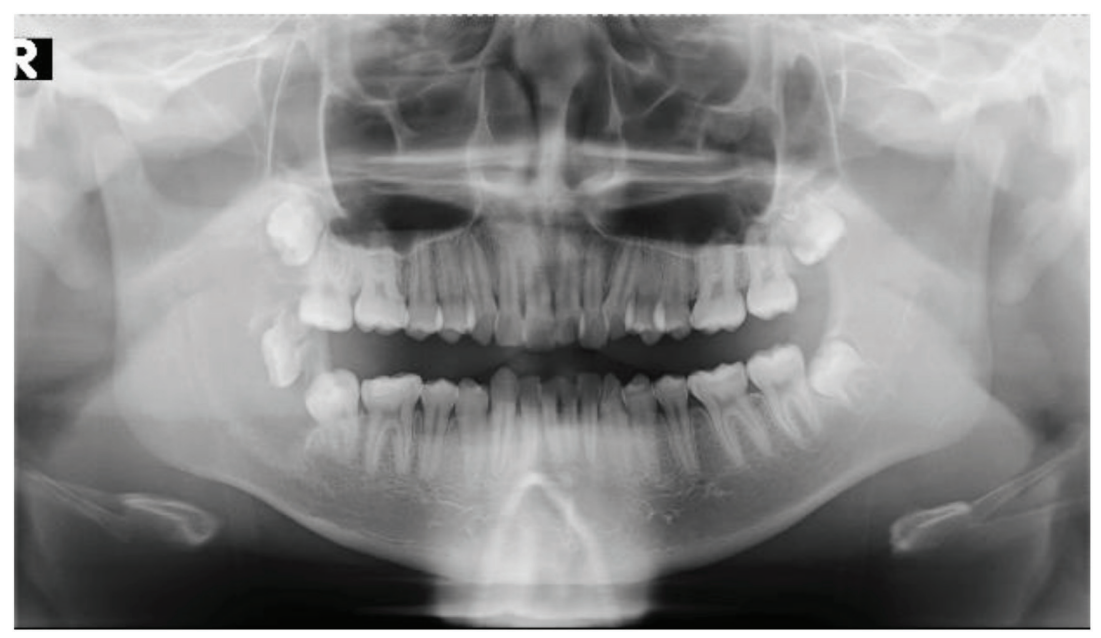

Figure 1 Panoramic radiograph shows unilocular radiolucent in the mandibular ramus and third molar was located above
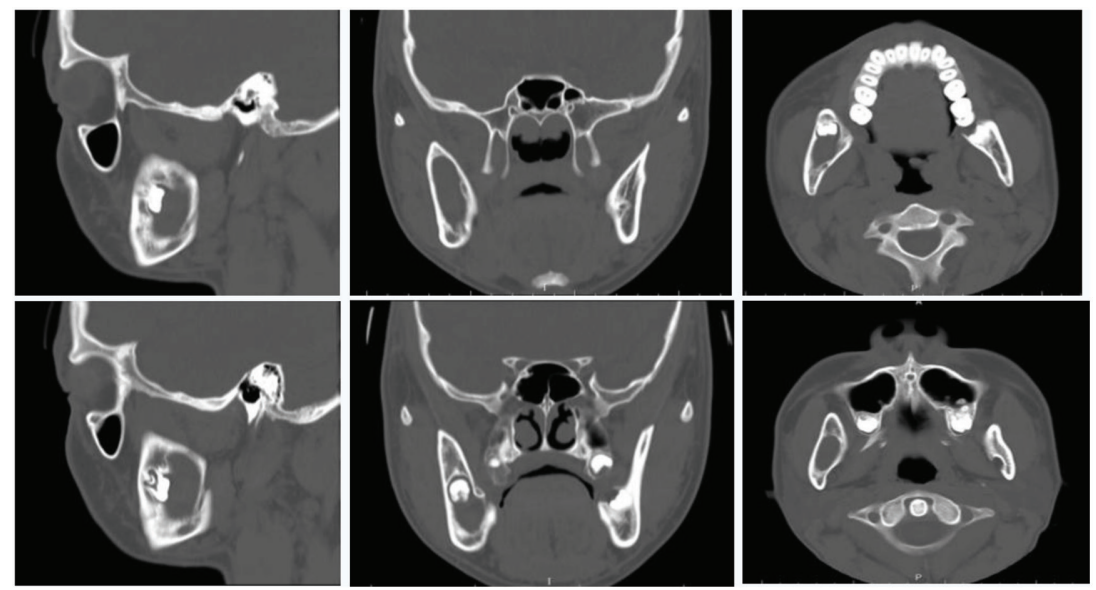

Figure 2 CT shows a cystic lesion including crown of third molar
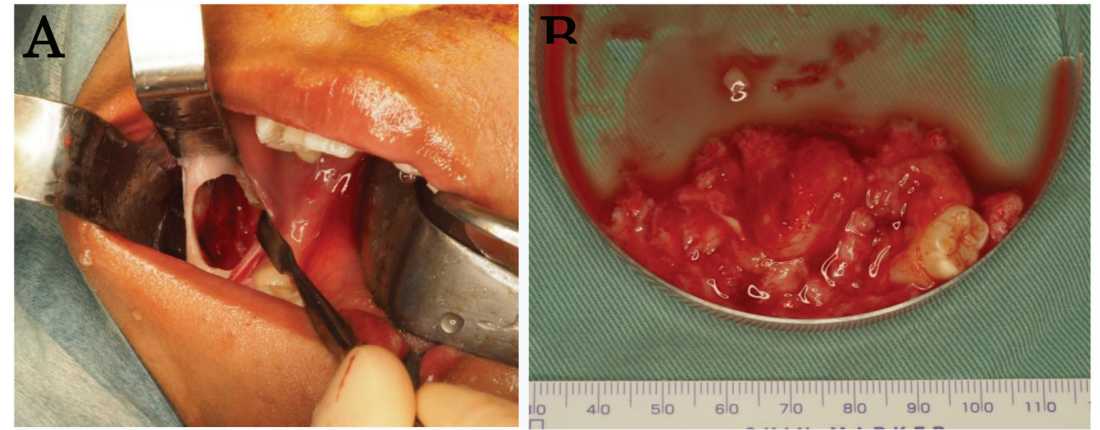

Figure 3 A. The lesion during enucleation, B. A photogtaph of the surgical specimen

\section{Discussion}

$\mathrm{AF}$ is an uncommon type of benign odontogenic tumor. It was classified as a mixed odontogenic tumorby the World Health Organization(WHO) in 2005because histologically AF consist of both epithelial and mesenchymalodontogenic tissue. ${ }^{1}$ Its incidence is approximately $1.5-4.5 \%$ of all jaw tumors. ${ }^{1,2,4,5} \mathrm{AF}$ is considered to be a tumor of

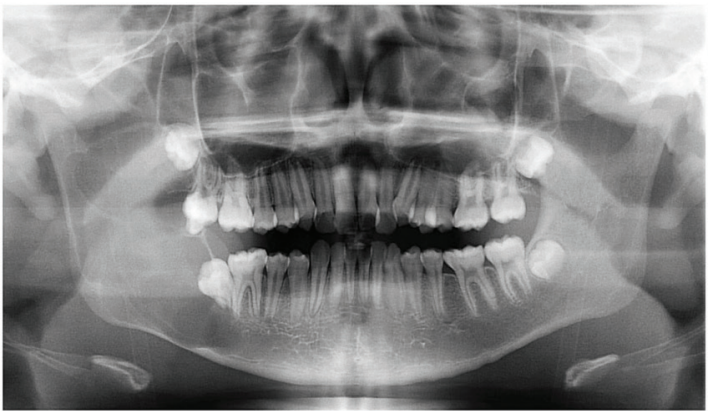

Figure 4 Panoramic radiograph 3 years ago.

An enlarge tumor occupy in the right mandibul ramus
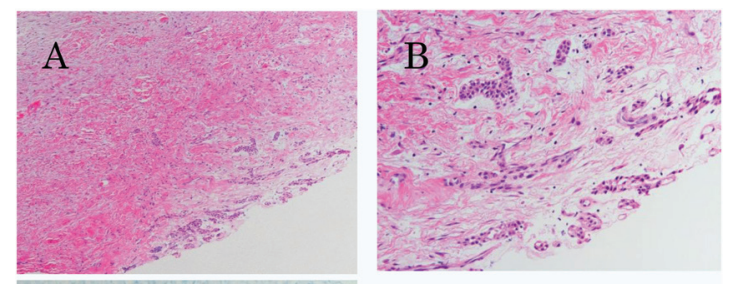

C

Figure 5 Histopathological examination. A-B: round islands and narrow cords of odontogenic epitheliumina cellular, primitive mesenchymalbackground $(\mathrm{H}$ E, $A[\times 40], B[\times 200])$. C: the Ki-67 index of the lesion was $0 \%$.

childhood and adolescence, occurs almost exclusively in the first and second decades of life. $\mathrm{AF}$ is a slow-growing benign tumorsoit does not exhibit characteristic clinical manifestations and is often detected incidentally during routine radiographic examinations. Usually, AF presents as a unilocular radiolucent lesion, but enlarging tumors can demonstrate a multilocular radiolucent pattern. $^{3}$

Some authors have reported that AF does not display a predilection for either sex. ${ }^{6}$ As for the tumor site, AF usually arises in the mandible, and the posterior mandible is affected more often than the maxilla by a factor of $3.1 .^{7}$

Among the 38 cases of AF reported in Japan, an impacted tooth was found within thelesion in 15 , and it has been suggested that $\mathrm{x}$-ray examinations might be useful for screening for $\mathrm{AF}^{8}$ As AF have smooth well-demarcated borders and present withunilocular or multilocular x-ray patterns, the differential diagnoses of AF include entities such as dentigerous cyst, ameloblastoma and keratocysticodontogenic 
tumor. Histologically, AF is a true mixed tumor, as it has both mesenchymal and epithelial neoplastic components, but does not contain any calcified tissue. ${ }^{9}$ Ameloblastic fibro-odontoma (AFO) is a tumor that shares many features with $\mathrm{AF}$, but alsocontains enamel and dentin. Some authors consider this lesion to be an intermediate stage in the development of odontoma, with the primary stage of formation being $\mathrm{AF}^{2}$ As some odontomas have similar histological features to AF and AFO, clinical findings are fundamental for differentiating among these three pathological entities. If all cases followed the developmental process outlined above, AF would affect young patients, odontoma would occur in elderly patients and AFO would be seen in an intermediate age group. However, this is not the case. In the past, AF was regarded as a variant of ameloblastoma,but it is currently considered to be of odontogenic epithelial origin.

The nature of $\mathrm{AF}$ remains enigmatic, and there has been a long debate as to whether AF represents a hamartomatous growth or a true benign neoplasm. ${ }^{3,6,7}$

In the present case, the lesion had reduced in size during the 3 years before surgeryeven though no treatment was administered during this period. In addition, it exhibited a Ki-67 index of $0 \%$. Therefore, it might have been ahamartoma.

Surgical excision or thorough curettage together with the removal of any affected teeth is the current treatment of choice for AF. The reported recurrence rate varies among sources, but is considered to be low. ${ }^{10}$

Trodahal et al. reported that 10 of $24 \mathrm{AF}$ recurred. ${ }^{11}$ Chen Y et al. ${ }^{4}$ found that 14 of 41 recurrent $\mathrm{AF}$ cases exhibited malignant transformation and the estimated 10-year malignant transformation rate was $25 \%{ }^{2}$ Two of the 38 cases of AF reported in Japan recurred and malignant transformation to ameloblastic fibro sarcoma occurred in one of these cases. ${ }^{12}$ We were not able to obtain any definitive histopathological evidence of bone neogenesis, but it is possible that bone neogenesis contributes to $\mathrm{AF}$ in cases in which the wisdom teeth are erupting.

\section{Conclusion}

Based on our experience we suggest that it is possible to follow-up young AF patients whose tumors do not exhibit any proliferative activity. However, clinicians should take the patient's age into consideration during treatment selection because cases of
AF involving patients aged $\geq 22$ years old are very likely to recur and undergo malignant changes.

\section{Conflict of Interest}

The author declare that they have no conflicts of interest.

\section{Acknowledgement}

We would like to thank Dr Fumishi Kunishima of the Department of Pathology Okinawa Prefectural Chubu Hospital for his help in preparing this manuscript.

\section{References}

1. Barnes L, Eveson JW, Shootweg PJ. World Health Organization Classification of Tumours Patholog \& Genetics Head and Neck Tumours. New York: WHO Publication Center; 2005. p. 308-309.

2. Chen Y, Wang JM, Li TJ. Ameloblastic fibroma: a review of published studies with special reference to its nature and biological behavior. Oral Oncol 2007;43: 960-969.

3. Poramate P-A, Andre C, Kittipong D, et al. Extensive ameloblastic fibroma in an adolescent patient: a case report with a follow-up of 4 year. Eur J Dent 2009;3: 224-228.

4. Chen Y, Li TJ, Gao Y, et al. Ameloblastic fibroma and related lesions: a clinicopathologic study with reference to their nature and interrelationship. J Oral Pathol Med 2005;34: 588-595.

5. Cohen DM, Bhattacharyya I. Ameloblastic fibroma, ameloblastic fibro-odontoma and odontoma. Oral Maxillofac Surg Clin North Am 2004;16: 375-384.

6. Amos B, Marilena V. Ameloblastic fibroma: a stage in the development of a hamartomatousodontoma or a true neoplasm? Critical analysis of 162 previously reported cased cases plus 10 new cases. Oral Maxillofac Pathol 2013;116: 598-606.

7. JindalC, Bhola RS. Ameloblastic fibroma in six-year-old male: hamartoma or a true neoplasm. J Oral Maxillofac Pathol 2011;15: 303-305.

8. Keiko Y, Yasunori N, Akihiko M, et al. A case of mesenchymal component-rich mandibular ameloblastic fibroma. Nihonkoukuugekashi 2011;57: 314-318.

9. Costa DO, Alves AT. Maxillary ameloblastic fibroma: a case report. Braz Dent J 2011;22: 171-174.

10. Brenda L, Gretchen S. Ameloblasticfibroma. Head and Neck Pathol 2009;3: 51-53.

11. Trodahl JN. Ameloblastic fibroma. A survey of cases from the Armed Forces Institute of Pathology. Oral Surg Oral Med Oral Pathol 1972;33: 547-548.

12. Kazuo K, Ryuichi M, Tohru F, et al. Malignant transformation of ameloblastic fibroma to ameloblasticfibrosarcoma: a case report and review of the literature. J Craniomaxillofac Surg 2005;33: 352-355.

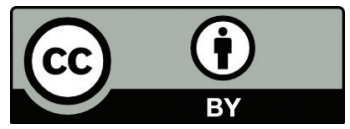

This work is licensed under a Creative Commons Attribution 